\title{
OFDM parameter design of a digital broadcasting system in FM band
}

\author{
Li Jilong, Yin Yanbin \\ Academy of broadcasting science, Beijing, China \\ lijilong@abs.ac.cn, yinyanbin@abs.ac.cn
}

\begin{abstract}
A thorough OFDM parameter design method of a digital broadcasting system in FM band is presented in this paper. Key OFDM parameters are analyzed from their constraints. Cyclic prefix should satisfy the requirement of channel delay and formation of SFN. Design of system bandwidth should consider coherent bandwidth and existing frequency planning. Symbol duration is restricted by time selectivity and frequency selectivity of the channel, and also efficient SINR should be maximized. System simulation is performed to test and verify the parameters under typical FM band channels. A set of OFDM parameter is provided for future design.
\end{abstract}

Keywords- digital broadcasting system; cyclic prefix; symbol duration; bandwidth

\section{INTRODUCTION}

Broadcasting systems now change from conventional analogue transmission to digital transmission. Digital audio broadcasting can provide higher spectral efficiency and power efficiency, and have better performance than analogue broadcast in multi-path channels. Digital audio broadcasting systems, such as DAB, DRM or HD Radio have been developed and applied in some regions.

FM band in VHF frequency is one main band for audio broadcasting. DAB and DRM is not designed for audio broadcasting in FM band until DRM+ is publicized in 2009. $\mathrm{DRM}+$ is compatible with DRM, which is designed decades ago. DRM+ uses the same system architecture as DRM, such as convolutional code, multiplexing and audio frame structure. FM HD-Radio works in FM band, but both laboratory and field tests prove it does not offer the audio quality and signal robustness performance that listeners would expect. And the low digital signal power level, which is $23 \mathrm{~dB}$ below the power of FM carrier, means that coverage of HD Radio is far below than that of the analogue [1].

Techniques change greatly in recent years. With the application of new techniques, better system can be designed, which can be expected to lower receiver's cost and improve system performance. In order to use the latest technology developed in recent years, a new digital audio broadcasting system is to be designed. In the design work, techniques, such as LDPC code and transmit diversity, are to be used. But first of all, OFDM parameters should be designed to guarantee a good performance in FM band channels.

In this paper, topic related to OFDM parameter design of a digital broadcasting system is presented. The topics presented here cover design of cyclic prefix length, symbol duration length and system bandwidth. System design includes more topics than what presented here, such as choice of modulation format, coding scheme, pilot pattern design, but they are out of the scope in this paper.

There are papers on optimization of single parameter of OFDM, such as guard interval length, sub-carrier bandwidth, symbol duration and pilot pattern respectively [2][3]. In [4][5], optimum values of timing offset, number of carriers and guard time duration of OFDM system on frequencyselective time-selective fading channels is analyzed based on degradation of SNR, it give the conclusion of how parameters change with other variables. Reference [6] gives a symbol length design method in terms of efficient symbol length for maximizing the effective SINR, and the method omits the influence of inter-symbol interference (ISI).

The related work mainly covers single parameter optimization of OFDM in communication system design. The work in this paper includes design of multiple OFDM parameters to form a prototype of broadcasting system, which has its unique characters. In broadcasting system, cyclic prefix length has a direct relationship with transmitter coverage and the formation of single frequency network (SFN). And the bandwidth of digital system has to comply with existing frequency planning for analogue broadcasting, and also can be extended for future all digital broadcasting. Therefore broadcasting system needs particular consideration in design process.

This paper is organized as follows. In section 2, OFDM parameters and multi-path channels in FM band are briefly reviewed. In section 3, analysis of OFDM parameters is made from channel and system requirement. Section 4 performs simulation of system parameters. In section 5, a prototype of digital broadcasting system is proposed. And paper is concluded in section 6 .

\section{SYSTEM DESCRIPTION}

As shown in Fig.1, OFDM symbol duration $T_{S}$ is divided into two parts: the effective symbol length $T_{\text {eff }}$ and the cyclic prefix length $T_{C P}$. Cyclic prefix is a repetition of the signal samples and used to mitigate the effect of time dispersion. When cyclic prefix length is longer than the duration of channel impulse response, ISI can completely be removed.

The sub-carrier bandwidth $\Delta f$, which is the inverse of the effective symbol duration, and is defined by

$$
\Delta f=1 / T_{\text {eff }}=1 /\left(T_{S}-T_{C P}\right)
$$

The system bandwidth can be found by the number of carriers $N$ multiply the sub-carrier bandwidth

$$
B_{S}=N \times \Delta f
$$




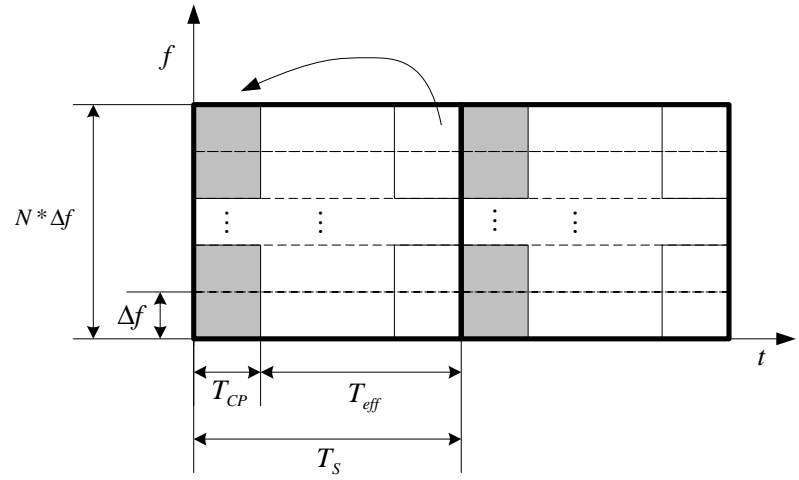

Figure 1. OFDM parameter description

The selective-fading channel in FM band in this paper is modeled by a number of delayed and attenuated flat-faded Rayleigh paths. It's the result from multi-path characterization tests in Salt Lake City conducted by EIA in 1993 and USADR has adopted this channel model. Four different multi-path models are used: "urban slow," "urban fast," "rural fast," and "terrain-obstructed fast." The "fast" and "slow" modifiers refer to the ground speed of the vehicle on which the receiver is mounted. This ground speed directly determines the degree of Doppler spread experienced by the signal. The fading scenarios are summarized in [7].

\section{ANALYSIS OF OFDM PARAMETERS}

\section{A. Design of cyclic prefix}

In broadcasting system, cyclic prefix length of OFDM system is constrained with the following restrictions:

1) To ensure that the OFDM symbols are not subject to inter-symbol interference, cyclic prefix length should be longer than maximum path delay, or longer than 4 times path delay spread [8]. The maximum path delay are 3us, 3us and 16us and the delay spread are 0.78us, 0.43us, 5.1us respectively in urban, rural and terrain-obstructed channels. Based on the above requirements, cyclic prefix length is at least 20.4us, which is 4 times the delay spreads in terrainobstructed channel.

2 ) In places where require broadcast network cover large areas or dense urban areas, single frequency network can be established. In SFN, several transmitters simultaneously send the same signal over the same frequency channel. SFN can reduce required transmission power, and increase broadcast coverage at the same time. At the receiver, time delay between received signals from different transmitters should be within cyclic prefix length of OFDM to get diversity gain of SFN. On the other hand, broadcasting system requires cyclic prefix length to satisfy the expected coverage of transmitter. Depending on operational requirements, transmission time of distance between transmitters should be 1.2 times cyclic prefix length of OFDM [9]. For a desired distance of $50 \mathrm{~km}$ between transmitters, cyclic prefix length larger than 139us is preferred.
From the above analysis of delay spreads and formation of SFN, OFDM cyclic prefix should be greater than 139 us.

Cyclic prefix can reduce ISI caused by the time dispersive channel. During cyclic prefix, no information can be transmitted, so there is an energy loss. The energy loss is more evident when the relative ratio between cyclic prefix and symbol duration is high. Therefore cyclic prefix length must be chosen sufficiently small.

\section{B. Design of symbol duration (sub-carrier bandwidth)}

Selection of OFDM effective symbol duration can be analyzed from the characteristics of transmission channel. The FM band channel is a time-selective frequency-selective channel, and choice of OFDM system parameters has to make a compromise between channel frequency selectivity and time selectivity.

From equation (1), if $T_{\text {eff }}$ is large, $\Delta f$ will be small. The level of inter-channel interference (ICI) is dependent on the Doppler frequency and frequency offset in relation to the sub-channel bandwidth. ICI increases when $\Delta f$ decreases, therefore it is important to choose $\Delta f$. This can also be explained from the view of time varying characteristic of channel. Symbol duration should be less than coherence time of the channel to reduce the effect of time varying channel on OFDM symbol. When time-dependent correlation is 0.5 , coherence time is defined by

$$
T_{c} \approx \frac{9}{16 \pi f_{m}}
$$

where $f_{m}$ is the maximum Doppler frequency offset, $f_{m}=f * v / c, v$ is the relative velocity between receiver and transmitter and $f$ is the transmitted frequency, $c$ is the velocity of light. For digital broadcast in FM band, the maximum frequency is $108 \mathrm{MHz}$ and the fastest expected receiver speed is $300 \mathrm{~km} / \mathrm{h}$, so the maximum possible coherence time is $6 \mathrm{~ms}$.

Symbol duration should be selected to ensure that OFDM sub-carrier bandwidth is less than coherence bandwidth, otherwise channel of sub-carrier would be frequency selective and signal may not be recovered by channel equalization. When frequency-dependent correlation is 0.9 , the coherence bandwidth can be defined as

$$
B_{c} \approx \frac{1}{50 \delta_{\tau}}
$$

where $\delta_{\tau}$ is the delay spread. The coherence bandwidth in urban, rural, terrain-obstructed environment are $25.6 \mathrm{kHz}$, $46.5 \mathrm{kHz}, 3.9 \mathrm{kHz}$ respectively, whose symbol period are 39.1us, 21.5us, 256.4us. The symbol duration should be greater than the largest symbol period 256.4us from analysis of coherence bandwidth.

From the above analysis of frequency selectivity and time selectivity of the channel, OFDM symbol duration should be within the period of 256.4us and 6ms.

\section{Design of system bandwidth}

The OFDM signal can not be received when signal fully falls into a serious fading. To avoid this, system bandwidth 
should be greater than coherence channel bandwidth. When system bandwidth is large enough, channel fading or narrowband interference can only affect a portion of OFDM sub-carriers, but cannot jam the entire radio link, and the signal may be recovered by digital processing at the receiver. Thus system bandwidth should be larger than $46.5 \mathrm{kHz}$, which is the maximum coherence bandwidth in FM channels.

It can be analyzed from the point of transmission theory [9]. Channel's transfer function $H(\omega)$ can be defined as

$$
H(\omega)=\sum_{i=1}^{n} a_{i} e^{-j w t_{i}}
$$

$a_{i}$ and $t_{i}$ represent attenuation and delay of the i-th paths. In multi-path channel, $P_{r}$ is the power of received signals from all paths, $P(\omega)$ is power at frequency $\omega$. $P(\omega) / P_{r}$ can be represented by the following formula

$$
P(\omega) / P_{r}=|H(\omega)|^{2} / \sum_{i=1}^{n} a_{i}^{2}
$$

In certain bandwidth $\Delta_{\omega}$, transmission power can be expressed as

$$
P / P_{r}=\left(1 / \sum_{i=1}^{n} a_{i}^{2}\right) \cdot \int_{-\Delta_{\omega} / 2}^{\Delta_{\omega} / 2}|H(\omega)|^{2} d \omega
$$

It can be seen that in a narrowband system, transmission power fluctuates greatly with variety of transfer function $H(\omega)$. If system bandwidth is wider, transmission power will be steady, and transfer performance will be the better.

\section{Parameter of existing digital system in FM band}

ITU recommends two digital audio broadcasting systems designed to work in FM band, DRM + and HD Radio. Both of them use OFDM technology. System parameters of DRM+ and HD Radio are shown in Table I $[10,11]$. It can be seen that the parameters of two digital systems all fit the result of above analysis.

\section{E. Analysis of symbol duration with maximum SINR}

Effective symbol duration can be analyzed in term of maximizing signal to interference and noise ratio (SINR) [6]. For simplicity, ISI is not considered in this SINR analysis, and only ICI is taken into account. The instantaneous SINR for a sub-carrier can be defined as

TABLE I. PARAMETER OF DIGITAL SYSTEM

\begin{tabular}{|c|c|c|}
\hline \multirow{2}{*}{ Parameter } & \multicolumn{2}{|c|}{ Parameter Value } \\
\cline { 2 - 3 } & HD Radio & DRM+ \\
\hline Cyclic prefix length(us) & 150.5 & 250 \\
\hline $\begin{array}{c}\text { Effective symbol duration } \\
\text { in standard (ms) }\end{array}$ & 2.748 & 2.25 \\
\hline $\begin{array}{c}\text { Sub-carrier bandwidth } \\
\text { (Hz) }\end{array}$ & 363.4 & 444.444 \\
\hline \begin{tabular}{c} 
System bandwidth (kHz) \\
\hline
\end{tabular} & $69 \times 2$ & 96 \\
\hline
\end{tabular}

$$
\rho_{\text {eff }}=\beta \cdot \frac{|h|^{2} \cdot P_{s}}{P_{I C I}+N_{0}}=\frac{T_{\text {eff }}}{T_{\text {eff }}+T_{C P}} \cdot \frac{|h|^{2} \cdot P_{s}}{P_{I C I}+N_{0}}
$$

where $\rho_{\text {eff }}$ is the effective SINR, $P_{s}$ is the transmitted power including that of cyclic prefix, $N_{0}$ is the noise power. $h$ is instantaneous channel gain factor of the sub-carrier,. The effective SINR should be calculated in average of the equation (9)

$$
\begin{aligned}
\overline{\rho_{\text {eff }}} & =\frac{T_{\text {eff }}}{T_{\text {eff }}+T_{C P}} \cdot \frac{\delta_{h}^{2} \cdot P_{s}}{P_{I C I}+N_{0}} \\
& =\frac{T_{e f f}}{T_{\text {eff }}+T_{C P}} \cdot \frac{P_{s}}{P_{I C I}+N_{0}}
\end{aligned}
$$

where $\delta_{h}^{2}$ is the normalized channel gain. ICI upper bound meets

$$
\frac{P_{I C I}}{P_{e}} \leq \frac{1}{6}\left(\pi f_{d} T_{e f f}\right)^{2}
$$

and with assumption of $P_{e}=\beta \cdot P_{s}$, the lower bound of the effective SINR can be obtained

$$
\overline{\rho_{\text {eff }, L}}=\frac{1}{\frac{1}{6}\left(\pi f_{d} T_{e f f}\right)^{2}+\frac{1}{\beta \cdot \gamma_{s}}}
$$

where $\gamma_{s}$ is the SINR of the symbol. It is found that the effective SINR can approach to zero when $T_{\text {eff }}$ goes to either zero or infinite. So there is a maximum effective SINR. By calculating the derivative of equation (11), the optimum $T_{\text {eff }}$ can be obtained.

$$
T_{\text {SINR,opt }}=\left(\frac{3 T_{C P}}{\left(\pi f_{d}\right)^{2} \cdot \gamma_{s}}\right)^{\frac{1}{3}}
$$

where $T_{\text {SINR,opt }}$ is the optimum efficient symbol duration for maximizing the effective SINR.

Using maximum SINR method, symbol duration of HD Radio, DRM+ are calculated, where $f_{d}$ is set to $15 \mathrm{~Hz}$ as in typical EIA channel, and $\gamma_{s}$ are referred to the references and are $13 \mathrm{~dB}$ and $15 \mathrm{~dB}$ for HD Radio and DRM+. The calculated effective symbol durations are $2.17 \mathrm{~ms}$ and $2.20 \mathrm{~ms}$ respectively for HD Radio and DRM+.

There are differences between symbol duration in standards and that from maximum SNR method. This is due to the assumptions in maximum SINR analysis does not completely comply with the facts. Meanwhile, system design is a combination of many factors, and can not take maximizing efficient SINR as a single goal. The analysis result from maximum SINR cannot be used directly and only be a reference in system design.

For the designed system, SINR threshold is assumed to be $15 \mathrm{~dB}$, which is same to that of DRM+. Cyclic prefix length is 150us, which is preferred to be selected in the designed 


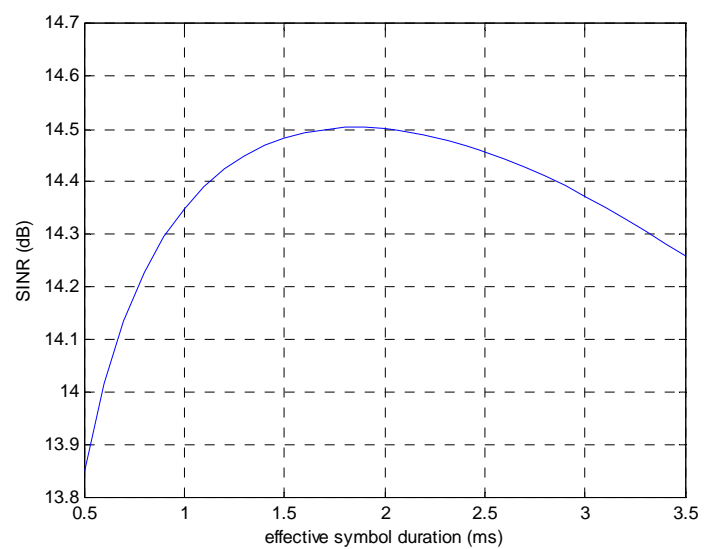

Figure 2. SNR vs. efficient symbol dutation

system, effective SINR is calculated with different symbol durations from formula (12) and shown in Fig.2. Maximum SINR is achieved when symbol duration is $1.857 \mathrm{~ms}$. Shorter or longer symbol duration will cause loss of effective SINR.

From channel characteristics and SINR maximization, OFDM parameters of digital broadcasting system in FM band have been analyzed and obtained, but theoretical analysis can not fully reflect the requirements of a real system. The analysis results need to be tested further in system simulation, which will be the content of next section.

\section{SIMULATION OF OFDM PARAMETERS}

In order to verify the performance of OFDM system parameters, system simulation has to be performed. In the simulation, digital system using a 1/2 rate convolutional code, whose constraint length is 7 , and the generator polynomial is (133, 171). Random interleaving is performed after channel coding, QPSK modulation is used, and rectangular pilot is inserted into the OFDM signal. Simulation channels are typical rural, urban, terrain-obstructed channels from EIA test. Least square channel estimation algorithm is used at the receiver.

\section{A. Simulation of system bandwidth}

Simulation is performed to analyze system performance under different system bandwidth. Sub-carrier number is selected to provide system bandwidth from $22 \mathrm{kHz}$ to 159 $\mathrm{kHz}$, and symbol duration is fixed at $1.93 \mathrm{~ms}$, which is within the range of previous analysis and near the result of maximum SINR analysis.

It can be seen from Fig. 3, when system bandwidth is narrower than coherent bandwidth, BER worsens with the decrease of bandwidth. When bandwidth is greater than coherent bandwidth, BER fluctuates in a small level. The simulation results show that system with narrower bandwidth can be easily inflected by channel fading or narrow band interference, and can not be normally recovered at the receiver.

The current channel spacing in FM band is $100 \mathrm{kHz}$ in ITU Region 1, 2 (Europe, Asia) and $200 \mathrm{kHz}$ in ITU Region

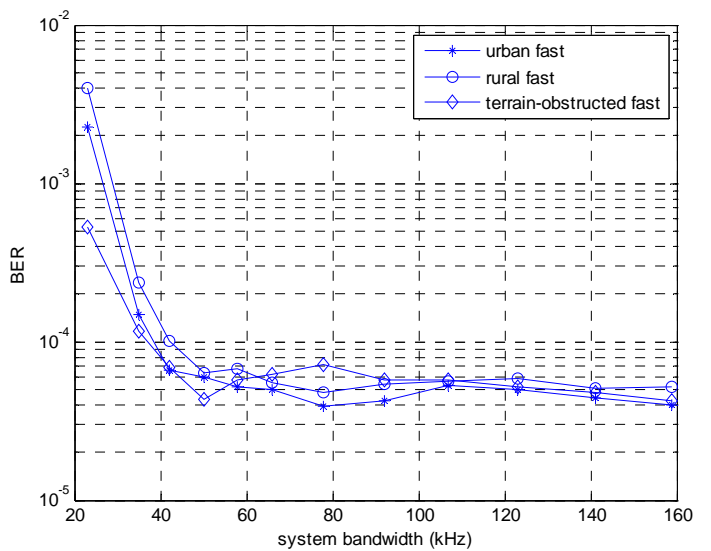

Figure 3. BER and System bandwidth curve with symbol duration $1.93 \mathrm{~ms}$

2 (North and South America). To make the transition from analogue FM broadcast to digital broadcast smoothly, digital system bandwidth should maintain compatibility with existing frequency planning for analogue broadcasting and is set to $100 \mathrm{kHz}$. And bandwidth can be extended to $200 \mathrm{kHz}$ or more by combination of multiple $100 \mathrm{kHz}$ bands. This kind of bandwidth setup can support high data rate when analogue broadcasting is no longer needed and all digital broadcasting is set up in future.

\section{B. Simulation of symbol duration}

To find suitable symbol duration, system simulation is performed. Different symbol durations are selected within the range of $0.81 \mathrm{~ms} \sim 3.05 \mathrm{~ms}$, which is $0.81,1.13,1.29,1.61$, 1.93, 2.09, 2.41, 2.73, 3.05ms. System bandwidth is fixed at $100 \mathrm{kHz}$. SNR threshold is kept at $15 \mathrm{~dB}$. Simulation is performed under typical EIA channels.

The simulation results are summarized in Fig.4. It shows change of BER with symbol duration under different channel environment. In terrain-obstructed channel, BER worsens with decrease of the symbol duration. When the symbol duration is shorter than $1.61 \mathrm{~ms}$, the BER is very high. In rural channel, the BER achieves the minimum value in the range of $1.61 \mathrm{~ms}$ and $1.93 \mathrm{~ms}$. When symbol duration is larger

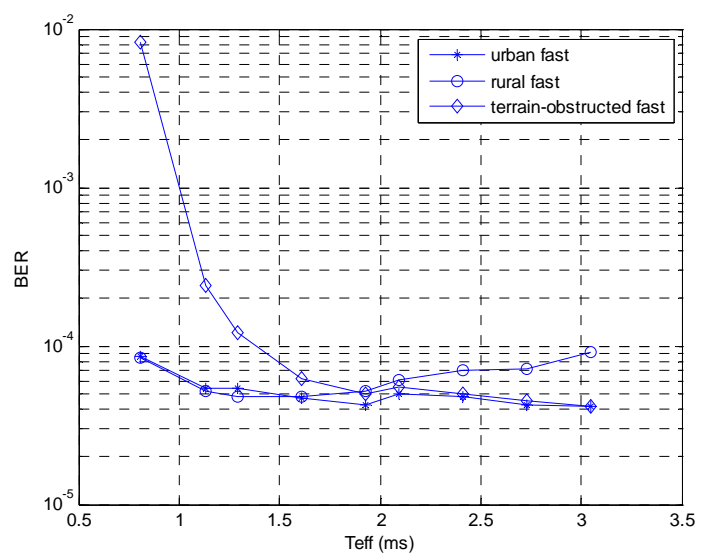

Figure 4. BER and symbol duration curve under typical channel 
than $2.5 \mathrm{~ms}$, the narrower carrier bandwidth is more easily influenced by Doppler shift and frequency offset, and BER increases with the increase of symbol duration. BER in urban channel fluctuates in a small level with all symbol durations.

From simulation of symbol duration under typical channels, it can be seen that when symbol duration is set within the period of $1.5 \mathrm{~ms}$ and $2.7 \mathrm{~ms}$, the digital system can be guaranteed to achieve a good BER performance in all channels. By system simulation, the possible range of symbol duration is shortened from the analysis result in section III.

Taking into account of the factors not considered here, such as, the system bandwidth, frame size, code rate, etc., symbol duration may be adjusted near the result of simulation analysis.

\section{DESIGN OF SYSTEM PARAMETERS}

In the parameter design, more aspects should be considered than what mentioned above. Some but not all of them are listed bellow

(1) Length of transmission frame is integer multiple that of audio frame, and time of audio frame should contain integer number of OFDM symbol period.

(2) From the view of the transmission efficiency, the OFDM symbol period should be large if allowed.

(3) Parameter setting should be conducive to the selection of clock frequency in the system.

From the analysis and simulation in this paper, a set of OFDM system parameters are got and shown in Table II.

TABLE II. PARAMETER OF DIGITAL SYSTEM

\begin{tabular}{|c|c|}
\hline Parameter & Parameter Value \\
\hline Cyclic prefix length(us) & 157 \\
\hline Effective symbol duration (ms) & 2.51 \\
\hline Sub-carrier bandwidth (Hz) & 398.4375 \\
\hline Number of sub-carriers & 244 \\
\hline System bandwidth (kHz) & 97.2 \\
\hline
\end{tabular}

\section{CONCLUSION}

This paper presents design of OFDM parameters in FM band broadcasting system. Cyclic prefix length is analyzed from the character of multi-path channel and the formation of SFN. System bandwidth is studied based on coherent bandwidth and existing frequency planning. Efficient symbol duration is selected by time selectivity and frequency selectivity of the channel, also energy efficiency with change of symbol duration is considered to achieve maximum SINR. Then simulation is performed to verify the result of analysis. A set of parameters is given in conclusion and can be used for further digital broadcasting system design.

\section{REFERENCES}

[1] Wolfgang Hoeg, Thomas Lauterbach, Digital Audio Broadcasting, John Wiler \& Sons Ltd., 2009

[2] Fredrik Tufvesson, Torleiv Maseng, Optimization of sub-channel bandwidth for mobile OFDM systems, MMT'97, Melbourne, Australia, Dec. 1997

[3] Ori Landau and Anthony J. Weiss, OFDM guard interval:analysis and observations, ICASSP 2007, April 2007, pp93-96.

[4] Heidi Steendam, Marc Moeneclaey, Optimization of OFDM on Frequency-Selective Time-Selective Fading Channels, ISSSE 98, Oct 1998

[5] Heidi Steendam, Marc Moeneclaey, Analysis and Optimization of the Performance of OFDM on Frequency-Selective Time-Selective Fading Channels, IEEE Transc. on Communications, Dec 1999

[6] Ma Meng, Yang Yuli, Jiao bingli, The optimum symbol length design for wireless OFDM over fast fading channels, Acta Scientiarum Naturalium Universitatis Pekinensis, Vol.42 No.5, Sept.2006, pp.658661

[7] Robert D. Culver, P.E., Lohnes and Culver, NRSC-R56, EIA-CEG / DAR Subcommittee, WG-B / Laboratory Testing and SG-1 / VHF Channel Characterization Test - Final Report, www.nrscstandards.org

[8] R.V. Nee, R. Prasad, OFDM for Wireless Multimedia Communications, Boston, Artech House, 2000.

[9] Li Dong, Digital Audio Broadcasting, Beijing Broadcasting Institute Press, 2001

[10] Digital Radio Mondiale (DRM): System Specification, ETSI, 2009

[11] HD Radio Air Interface Design Description Layer 1 FM, iBiquity Digital Corporation, August 7, 2007 